\title{
Desbordamientos y viaje hacia la izquierda. Prehistoria del movimiento 15M: de \#Nolesvotes a Democracia Real Ya
}

\author{
Overflows and turn towards the left side. 15M movement's \\ prehistory: from \#Nolesvotes to Democracia Real Ya
}

ADRIANA RAZQUIN*

\begin{abstract}
Resumen: Este artículo tiene la misión de arrojar cierta nitidez sobre quiénes son los agentes, cuáles las organizaciones, las sinergias y encuentros que sostuvieron, lo que podemos denominar, la prehistoria del movimiento $15 \mathrm{M}$. Se explica la gestación de este movimiento popular estructurado sobre cuatro desbordamientos (de participantes y de ideas fuerza) y tres bloques centrales que alimentaron la plataforma Democracia Real Ya: \#Nolesvotes, Coordinadora Ciudadana y Juventud Sin Futuro.

Palabras clave: movimiento $15 \mathrm{M}$, antecedentes sociopolíticos, etnografía, composición sociopolítica.
\end{abstract}

\begin{abstract}
This article has the aim to inform about the agents, organization, synergy and meetings which contributed to shape what can be called the prehistory of the $15 \mathrm{M}$ movement. An explanatory system is exposed in order to examine the formation of this popular movement. This examination goes both through four crucial moments that arrived when the movement became overwhelmed by the arrival of new participants and leading ideas and through three central blocks that feeded the platform Democracia Real Ya: \#Nolesvotes, Coordinadora Ciudadana and Juventud Sin Futuro.
\end{abstract}

Keywords: $15 \mathrm{M}$ movement, socio-politic background, ethnography, sociopolitical composition.

\section{Marco de aproximación teórico-metodológica}

La tarea de rastrear hasta determinar con más o menos nitidez los antecedentes sociopolíticos de la convocatoria del 15 de mayo de 2011, que bajo el lema Democracia Real Ya. No somos mercancía en manos de políticos y banqueros, movilizó masivamente a la ciudadanía a las calles, resulta compleja y, a día de hoy, inconclusa. En ese sentido, la información que aquí

Fecha de recepción: 12/02/2013. Fecha de aceptación definitiva: 17/05/2014.

* Es Doctora e investigadora del Grupo El problema de la alteridad en el mundo actual HUM-536, Universidad de Cádiz. Entre sus principales líneas de investigación se encuentran la propuesta teórica y metodológica de la escuela de Pierre Bourdieu y de la Sociología Crítica española, los movimientos sociales, especialmente el 15M, y los estudios feministas. Recientemente ha defendido la tesis doctoral que lleva por título Tomar la palabra en el 15M: condiciones sociales de acceso a la participación en la asamblea. Un estudio de caso. Entre sus publicaciones más recientes destacan «En la plaza se habla. Algunas claves para analizar la toma de la palabra en el 15M», Ímago Crítica (4, 57-77), Anthropos, 2013; y «Apuntes para una recepción de La dominación masculina de Pierre Bourdieu entre el feminismo del Estado español», en J. G. Núñez Olguín, González Martínez, M. N. y Miranda Medina, C. F. (Coords.) Discurso, compromiso e historia. Una aproximación sociológica al trabajo intelectual y político, Universidad Simón Bolívar, Colombia, 2014. Contacto: adrianarazquin@gmail.com 
se presenta estará siempre abierta a ser ampliada y matizada a medida que los estudios empíricos sobre este cuerpo social se amplíen e intensifiquen, pues este artículo no puede sustituir a un trabajo histórico exhaustivo que resultaría muy pertinente. Por el contrario -y asumiendo el riesgo- se inspira en la recomendación bourdisiana de esbozar, al menos, aquellos elementos que pertenecen al campo definido como objeto de estudio (Bourdieu y Wacquant, 2005, 324). $\mathrm{Y}$ en ese sentido, es la primera parte de un estudio socioetnográfico de una asamblea del 15M, que ha tenido como objeto explicitar las condiciones sociales de acceso a la toma de la palabra.

La etnografía de la que nace el trabajo que aquí se presenta se ha desarrollado en la asamblea general (junto con la acampada y todas las asambleas de barrios y pueblos y comisiones que nacen de ella) de una capital de provincia andaluza mediante observación participante de un año y un trabajo sobre relatos de vida (Bertaux, 2005 y Bourdieu, et al., 2010) reconstruidos mediante entrevistas abiertas semidirectivas (Ortí, 1986 y Bourdieu y Wacquant, 2005).

En concreto, los datos empíricos que se movilizan en este artículo provienen de 42 entrevistas abiertas (de las cuales 38 pertenecen a la reconstrucción de trayectorias y el resto a informantes, la mayoría participantes de Asamblea Sol), de la observación participante durante un año, conversaciones informales, $y$, de manera preponderante, del análisis de manifiestos, documentos de trabajo, actas de la asamblea general y de asambleas barriales, comunicados, filmaciones audiovisuales, documentales (editados), entrevistas en prensa y ensayos de opinión.

A partir de la articulación analítica del concepto de campo político de Bourdieu (2000), sitúo al movimiento $15 \mathrm{M}$ en dos momentos. Primero, como movimiento popular, donde la característica principal de este momento es que se involucra en las tareas organizativas y decisorias a multitud de profanos respecto del mundo político -no especialistas de la militancia o de la política-. Y, segundo, como un movimiento social ${ }^{1}$, donde puede diferenciarse entre la organización de la vida militante y toma de decisiones (a cargo de militantes a tiempo completo) y el cuerpo social que se moviliza con él.

1 A partir del trabajo de Gerard Mauger (2003, 33-37; 2013, 25-31), sitúo a esta segunda fase del 15M (como movimiento social) dentro del campo político, en un espacio más o menos periférico.

Respecto a la discusión entre Mauger y Mathieu sobre la conceptualización de los movimientos sociales en su relación con el campo político, Lilian Mathieu construye el «espacio de los movimientos sociales», que describe como un universo práctico y de sentido autónomo al interior del mundo social (Mathieu, 2004, 33) y en el seno del cual las movilizaciones están unidas por relaciones de interdependencia (Mathieu, 2007, 134). Mauger se ha opuesto a esta conceptualización al sostener que los movimientos sociales forman parte del campo político y que se situarían en el polo izquierdo del mismo. Según Mauger, forma parte de la lucha en el seno del campo político la misma delimitación de las fronteras del movimiento social. Y, en ese sentido, no puede hablarse de «espacio de los movimientos sociales» porque no presenta un grado de objetivación, de estructuración y de institucionalización suficiente, capaz de corresponderse con la definición de campo de Bourdieu (Mauger, 2003, 33-37). Una buena presentación de la discusión entre Mathieu y Mauger, junto con una exposición de otras aproximaciones resonantes con el planteamiento de Mathieu (Melucci, 1985, 99; Mc Carthy y Zald, 1977, 1220 en Mathieu, 2007, 134-135; Pechú, 2001, 73-74; Poliak, 2008, 78) excede los límites de este pie de página. Pero sí se debe señalar que, a pesar de que de cara a una conceptualización que de cuenta de la pluralidad ideológica presente en los distintos tipos de movimientos sociales contemporáneos, la aproximación de Mathieu resulta más adecuada para aprehender la diversidad, pues difícilmente se pueden reducir todos los movimientos sociales a la izquierda del campo político, como propone Mauger; para el trabajo que se presenta la aproximación de este último resulta adecuada. Pues, a pesar de la diversidad y pluralidad ideológica con que nació el movimiento 15M, (que aquí estamos presentando), el paso a movimiento social incorporó, al mismo tiempo, una resituación ideológica del proceso. Así, el 15M en la fase de movimiento social puede situarse claramente a la izquierda del campo político. Tanto por la redefinición de los fines últimos de la lucha, como por la batería de prácticas movilizadoras que se sostuvieron y por el tipo de organizaciones con las que se establecieron alianzas. 
La forma de movimiento social se va gestando poco a poco a lo largo de las tres primeras semanas de acampada -para estar operativo en el momento en que se desmonta la acampada, aunque su consolidación definitiva se pueda situar en el otoño de 2011- a partir de los esfuerzos más o menos inconscientes de multitud de agentes que orientan la práctica y el discurso hacia una «politización» del movimiento, incorporando una lógica de acción que, en los primeros días, estaba completamente vetada: el análisis y contenido en clave política. Al mismo tiempo, las demandas de concreción y uniformidad de fines abanderadas por representantes de distintas facciones del centro del campo político (políticos profesionales y periodistas principalmente) y la propia necesidad de dar respuesta a demandas del contexto (elaboración de documentos, la gestión con la policía, el contacto con periodistas o abogadas, por ejemplo) van construyendo, en primer lugar, un espacio de legitimidad y prestigio para los y las agentes implicados en la tarea -lo primero que comienza a prestigiarse es el capital militante (Poupeau, 2007, 10)-. Y, después, para lo estructural del sentido de sus acciones: una lógica de acción y evaluación que no es espontánea, que ha de ser educada y, por tanto, que no todos los agentes comparten: están quienes saben «jugar el juego» y quienes no comprenden la dinámica de los efectos (Bourdieu, 2000, 9-10).

Este artículo buceará en el primer momento (movimiento popular), específicamente en la antesala de la convocatoria del 15 de mayo, que fue el acontecimiento que cimentó el comienzo del movimiento popular: lo que he llamado «prehistoria» del movimiento 15M. En él pretendo rastrear el origen sociopolítico de todos los agentes (individuales y colectivos) que tomaron parte en el proceso de gestación de la convocatoria, desde la perspectiva de que en las ideas fuerza que se constituyeron y defendieron ya durante la gestación de la convocatoria, podemos encontrar el origen de los consensos y disensos que acontecieron en las asambleas, fundamentalmente, en ese primer momento como movimiento popular.

\subsection{Sociogénesis de un proceso vivo}

Esta reconstrucción es el punto de partida de la sociogénesis de un proceso vivo que, en el momento de redacción de este artículo, continuaba escribiendo su historia, definiendo y redefiniendo su mitología fundacional. Ofreciéndome la posibilidad de entrever, con cierta nitidez, la lucha por el establecimiento de las definiciones -el caso de las autonominaciones, la identidad colectiva, los fines últimos de la lucha- o los calendarios de hitos del movimiento (Bourdieu, 2012, 20-21). Esto es, me permitió etnografiar el acontecer de las luchas simbólicas involucradas en los procesos de construcción de una cosmovisión que se concreta, y se torna empíricamente accesible, a través de los elementos que componen el relato mitológico-histórico y sus implicaciones prácticas.

El trabajo de reconstrucción ha resultado complejo. Por un lado, el acceso a la información tenía que ser, por la naturaleza del fenómeno, en buena medida sostenido por el relato de informantes. Personas que participaron en el proceso de construcción, en las reuniones, en los debates y que en su mayoría comportaban posiciones de compromiso con la lucha en el campo político en el momento de la entrevista o habían salido del proceso tras una participación más o menos amarga. Una polarización política que venía gestándose en la acampada y que se imponía con fuerza sobre el proceso ya en el verano de 2011. Y esa lucha se jugaba (se juega aún de manera retrospectiva) fundamentalmente en el terreno de lo 
simbólico, donde «la fundación» es un elemento en disputa -en disputa, por tanto, los padres y las madres de la fundación- tendente a impregnar la narración de los acontecimientos de manera más o menos densa con las apuestas en la contienda simbólica.

Por otro lado, la velocidad y voracidad con la que se sucedieron los acontecimientos hace, de la experiencia situada de cada participante, una suerte de fragmento de un caleidoscopio que compone un cuerpo colectivo (y que presenta cierta facilidad para reordenarse en una figura ligeramente diferente), del que se conoce una parte, o unas cuantas partes. Existen demasiadas zonas oscuras, demasiadas comisiones en las que no se estuvo, demasiadas reuniones simultáneas, sucesos paralelos, respuestas colectivas improvisadas, etc.: es como si el fenómeno abordara y desbordara a los agentes.

\section{2. \#Nolesvotes}

A comienzos de febrero de 2011 las páginas webs relacionadas con el mundo del intercambio de archivos y la libre circulación de material audiovisual, posicionadas respecto a la discusión sobre el pago y cobro de los derechos de autoría y el intento legislativo de la ministra de cultura González Sinde, publicitaban una campaña que llamaba a no votar por el PSOE, el PP y CIU en las elecciones municipales y provinciales de mayo del mismo año. La propuesta se enmarcaba inicialmente en una posición contraria a la «Ley Sinde²» (de ahí que se pidiera el voto a cualquier otro partido diferente del que la había elaborado y de los otros dos que la habían apoyado) que estaba lista para votación y sobre la que los tres partidos seguían teniendo compromisos de aprobación. Se llamaba a un boicot de voto como castigo tratando de forzar movimientos de posiciones $\mathrm{y}$, de paso, demostrar que lo que estaba en juego tenía repercusiones en la vida política nacional; porque no solo eran cibernautas, también eran ciudadanos y ciudadanas. Trataban, pues, de situar su resistencia a la ley alejando el discurso del berrinche de quien goza descargando pelis y series rápido y gratis: gorrones a costa del deslomo de los artistas -como se les estaba calificando desde numerosos medios de comunicación e instituciones estatales-. Sostenían su oposición a la ley en el marco de una resistencia llamada a defender derechos civiles en el espacio virtual.

Las discrepancias a las modificaciones saltaron rápidamente del entorno cibernauta y la discusión copó los medios de comunicación -columnas, editoriales, reportajes, analistas, tertulias-, engarzándose además con un debate supranacional y teniendo repercusiones en las posiciones de los partidos políticos, como se verá en el devenir de los apoyos al paquete de medidas que incluía la Ley de Economía Sostenible o en la dimisión de Alex de la Iglesia, sustituto de Ángeles González Sinde al frente de la Academia de Cine, por discrepancias con la forma de gestionar el conflicto por parte de la ministra. Basta ver la noticia publicada por El País en su versión digital del veintiuno de diciembre de 2010, con el título «Ley Sinde, contra las cuerdas ${ }^{3}$ », que da cuenta de la dimensión política de la protesta cibernauta. Que indudablemente generó deslizamientos, cambios y afirmación

2 Utilizaremos este nombre para agrupar la idea legislativa - es importante señalar que como tal nunca ha sido aprobadade regular el cobro y pago de los derechos de autoría intelectual de los productos culturales en el espacio de Internet. Recibe el nombre de la ministra de cultura del PSOE encargada de dicha propuesta legislativa, Ángeles González-Sinde.

3 Noticia completa disponible en: http://elpais.com/diario/2010/12/21/cultura/1292886002_850215.html 
de posiciones con respecto a la regulación de las descargas en Internet; y, cómo no, de las fuerzas de movilización. En ese momento había entrado en acción Anonymous.

A unos días de la votación del paquete de medidas donde estaba incluida la regulación de las descargas en Internet, el viernes 17 de diciembre de 2010:

J. convocó una manifestación de protesta contra la legislación antidescargas en la plaza de la Constitución, de Málaga. Acudieron dos personas: J. y G. [...] Cuatro días después de aquella friquiconcentración con dos manifestantes y siete agentes del orden, J. trasladó el golpe a la red. [...] Convocó un evento de ataque en Facebook y entró en los canales IRC (Internet Relay Chat) para reclutar anons [miembros de Anonymous] que se sumaran al ataque. Unas 5.000 personas participaron en la operación. Cayeron las webs de los partidos. 'Ese es el poder de Internet: la difusión de una idea depende de que sea buena. Si lo que alguien dice tiene valor, la inteligencia colectiva lo eleva. Al día siguiente, los partidos no se atrevieron a apoyar la ley Sinde' [comenta J.]. (El País Domingo, con fecha de 5 de febrero de 2012: 10-11).

Quedaba inaugurada, entonces, la «ciber-acción», el «ciber-movimiento».

Pero retrotraigamos el relato un año antes; entonces las intenciones reguladoras de González Sinde ya eran conocidas. De hecho, su nombramiento como ministra estuvo salpicado de polémica en las redes sociales virtuales por sus declaraciones anteriores en calidad de presidenta de la Academia de Cine ${ }^{4}$.

La movilización contra la Ley Sinde ${ }^{5}$ había tenido origen en una campaña iniciada en la página web www.noalaleysinde.com donde se analizaba la ley y se llamaba a la movilización en el «Manifesto en defensa de los derechos fundamentales en Internet» ${ }^{6}$. Se hizo público, en diferentes páginas webs, el 2 de diciembre de 2009, el mismo día que el presidente del Gobierno presentaba el Anteproyecto de Ley de Economía Sostenible (dentro de las Disposiciones Finales se encontraban los elementos fundamentales de la llamada Ley Sinde).

Este manifiesto de finales de diciembre de 2009 estaba centrado en una oposición a la forma en la que el Gobierno -y sus socios- habían construido un mecanismo legal para regular los derechos de la propiedad intelectual en Internet, fundamentalmente hasta el punto seis.

4 Incluyendo en enero de 2011 la apertura de una petición de dimisión de la ministra en el portal www.actuable.es (este portal permite generar campañas de petición, iniciadas por personas particulares o grupos, publicitando la recogida de firmas que tienen validez únicamente como sondeo). La petición (que he datado según lo que recoge la prensa, pues el portal no ofrece la fecha en la que se hizo el llamamiento) fue firmado por 26.265 personas. Se acompañaba del siguiente texto: «Ministra Sinde: nosotros, los votantes, pedimos tu dimisión. Pido la dimisión de la ministra González Sinde por aprobar una Ley de espaldas al pueblo al que dice representar. El pueblo se ha manifestado en contra de esa ley, y, por lo tanto, como parte del pueblo he dejado de depositar mi confianza en la ministra. Además quisiera recordar al gobierno que no somos números en bancos, no somos monedas y billetes de euro. Somos personas, ciudadanos criminalizados, cuya libertad ha sido agredida por susodicha ministra. Como parte del pueblo que se ha manifestado mayoritariamente contrario a la Ley Sinde, pido la dimisión de la ministra por no defender los intereses de los ciudadanos, en pro de la democracia que los ciudadanos deseamos».

5 Con anterioridad venía gestándose una oposición fuerte al modo de protección a la propiedad intelectual escogido por el Gobierno (y apoyado por la mayoría de la Cámara). Véase el caso del «canon digital» y la respuesta liderada en aquel momento por la Asociación de Internautas, con Víctor Domingo a la cabeza.

6 Debido a que por cuestiones de espacio resulta imposible reproducir el manifiesto, invito a consultar el texto completo en: http://www.internautas.org/html/5871.html 
Parece que en la articulación del discurso de oposición se escoge un lugar político regido por lo que Andrés de Francisco $(2007,56)$ llama «corolarios prácticos del liberalismo dogmático». Primero, todo intento regulador o planificador de los poderes públicos es sospechoso o directamente perjudicial: «Internet debe funcionar de forma libre y sin interferencias políticas auspiciadas por sectores que pretenden perpetuar obsoletos modelos de negocio e imposibilitar que el saber humano siga siendo libre».

Acto seguido el texto dice: «Exigimos que el Gobierno garantice por ley la neutralidad de la Red en España, ante cualquier presión que pueda producirse, como marco para el desarrollo de una economía sostenible y realista de cara al futuro». La idea es que el Estado regule, contradictorio, en principio, con el primer corolario práctico. Sin embargo, considero que la clave reside en «sostenible y realista de cara al futuro», porque con eso se refieren a que es insostenible mantener el marco de los derechos de autor (arcaico) y que corre el riesgo de entorpecer el devenir de Internet. Es una defensa del Estado interesada y que se enmarca bien en las prácticas liberales economicistas: desaparición del Estado social y existencia de un Estado cuya única vocación sea asegurar los beneficios y las posibilidades prácticas a las empresas ${ }^{7}$. El Estado no debe interferir en la legalidad o no de los contenidos, su papel único es garantizar el «libre» mercado ${ }^{8}$. El Estado, escuálido, debe dejar hacer, debe dejar pasar, conceder el protagonismo a la sociedad civil (que está gobernada por la lógica del interés privado).

Segundo corolario, concepción aséptica y neutral de los mercados, que son lugares donde hay intercambios voluntarios sin poder ni coerción. Esto es, la economía se desconecta de la política. Y tercero, la política pasa a ser el espacio en el que los agentes, de manera individual y colectiva, defienden competitivamente sus intereses particulares (De Francisco, 2007, 57).

Pero el manifiesto también incide en otros elementos que comienzan a indicarnos que el discurso va más allá del debate sobre la libre circulación de información, o del pago/cobro de los derechos de autoría. En concreto, el manifiesto dedica el punto diez exclusivamente a reflexionar sobre la vertebración del mundo de los partidos políticos y la ciudadanía: la articulación, el diálogo (fundamentos para la buena representación parlamentaria) entre los representantes y los representados.

\subsection{Primer desbordamiento}

Esta incursión en el espacio de la crítica a la gestión política de los intereses públicos está ya consolidada, un año después, en la declaración de intenciones de la campaña \#Nolesvotes.

7 En el tercer párrafo contando por el final, se exige al Gobierno que «[...] garantice por ley la neutralidad de la Red en España, ante cualquier presión que pueda producirse, como marco para el desarrollo de una economía sostenible y realista de cara al futuro». Con esta exigencia, en el fondo, le confieren al Estado la tarea de garantizar que las reglas del juego neoliberal (el que puede, puede; el que se queda obsoleto, se queda obsoleto: reinvéntate o muere) tengan espacio para instalarse amplia y confortablemente. No se demanda la responsabilidad del Estado en la regulación de contenidos o en la gestión de Internet o en las condiciones de conexión, privacidad, accesibilidad, etc.; sino que se garantice, precisamente, que lo que este sector considera lobbys mediáticos (se refieren a todo el mundo de la Academia de Cine, actores, actrices, escritoras, ensayistas, etc. que forman parte del universo simbólico de la izquierda moderada, o la socialdemocracia identificada con el Gobierno del PSOE) dejen de tener poder para conseguir que se apruebe legislación que proteja los derechos de autoría, que son considerados un obstáculo para el libre desarrollo del potencial económico de una red de Internet sin regulación.

8 «La conclusión lógica es -en términos políticos- la necesidad de un Estado mínimo garante de las leyes contractuales basadas en la propiedad privada y proveedor de los bienes públicos estrictamente necesarios para el buen funcionamiento de los mercados» (De Francisco, 2007, 57). 
El tema se amplia, se intensifica la postura con respecto a la política nacional. La página principal del sitio web está titulada con «Ni PP ni PSOE \#nolesvotes» seguido por una imagen del mapa de España en el que están etiquetados los casos (resueltos o no judicialmente) de corrupción política de cargos públicos de diferentes partidos, localizados geográficamente. Clicando sobre cualquiera de las banderas de partidos se abre un cuadro de diálogo que explica brevemente las imputaciones y los cargos públicos implicados en cada caso junto con un enlace que lleva a la noticia. Le sigue un manifiesto9. En él, la oposición a la Ley Sinde es desbordada -hasta el punto de no quedar, apenas rastro de ella (se intuye al final del tercer párrafo «Los anteproyectos de ley»)- por una fuerte crítica al estado de las relaciones entre la «clase política» y la ciudadanía. De hecho, el texto arranca planteando la corrupción del sistema político, el clientelismo instalado en el partido-aparato («no llegan al poder los mejores, sino los que desde muy jóvenes se han dedicado a gestionar influencias e imponer la disciplina de la jerarquía») o la perversidad de la Ley electoral ${ }^{10}$, que impide una buena representación de la pluralidad ciudadana en las Cámaras. Para concluir que el Estado mismo está secuestrado por los intereses de potentes grupos de presión («minorías mediáticas o económicamente poderosas»). Un secuestro de las labores de gestión pública que no conoce límites: «Los anteproyectos de ley se elaboran a espaldas de los ciudadanos, manipulando la información y dando por buenos estudios e informes fraudulentos». En el marco de esta visión ciertamente orwelliana del Estado y las dinámicas sociales que acoge -cabría preguntarse: ¿a pesar de él?-, el texto explica que la única oportunidad de participación en una transformación de la esfera de lo público, es la convocatoria electoral. Pero aquí existe el problema de las listas cerradas y bloqueadas de los partidos que se alternan el poder: PSOE, PP, CIU, PNV-EAJ -cabría también preguntarse aquí: ¿es un mal único de estos cuatro partidos? ¿Es lo que los caracteriza por oposición al resto de formaciones políticas?-.

Llegados a este punto, el texto plantea dos opciones: «Puedes rendirte una vez más, hacerles caso. Pero también puedes tomar conciencia de tu poder como ciudadano: abrir los ojos e implicarte personalmente en la red de ciudadanos hastiados que consideran que está en nuestras manos mejorar la situación». Para ello \#Nolesvotes propone votar a cualquier otro partido que no sea uno de los mencionados más arriba, que «quizá representan mejor tus ideas», cerrando con la idea de que la democracia son los ciudadanos y las ciudadanas corrientes y no los «grandes partidos».

Encontramos aquí ya elementos característicos del primer manifiesto público de Democracia Real Ya, algunas de las ideas fuerza, pero no todas.

\subsection{Segundo desbordamiento}

Según cuentan Enrique Dans y Ricardo Adolfo Galli ${ }^{11}$ en el documental «\#nolesvotes \#democraciarealya Charla con Enrique Dans y Ricardo Galli», filmado en Mallorca el 18 de

9 Disponible en: http://www.nolesvotes.com/

10 Sobre los problemas representación de los partidos minoritarios por efecto de la Ley Electoral, así como sobre el contexto sociopolítico en la que se gesta, la Transición democrática tras la muerte del dictador, véase «Leyes electorales, sistemas de partidos y élites: el caso español» (Gunther, 1989, 77-101).

11 Enrique Dans es una de las personalidades más famosas cuando se trata el origen de Democracia Real Ya, o incluso, del 15M. Es profesor en sistemas de información en una escuela de negocios, licenciado en biología 
mayo de 2011, fue Carlos Sánchez Almeida, abogado especializado en contenidos digitales e Internet y autor del blog «Jaque perpetuo» para elmundo.es (espacio virtual del periódico de tirada nacional El Mundo), quién les manda una invitación para visitar un enlace:

[...] que no nos aclarábamos de lo que era, realmente, [Risas] era muy misterioso. Y, a partir de ahí, nos empezamos a reunir virtualmente [...] para escribir un primer manifiesto con la idea de: si han hecho esto... ¡No deberíamos votarles! Tendremos que votar a otros, porque lo que han hecho, vía la Ley Electoral y vía... Están montando un turnismo, un bipartidismo absoluto, en el cual han destruido el concepto que tenemos de democracia» (Enrique Dans, documental \#nolevotes\#democraciarealya).

Como expliqué más arriba, el desbordamiento de discurso es evidente: hablar de la Ley Sinde es hablar de la Ley Electoral, de «bipartidismo» y de corrupción política.

Detengámonos un instante y volvamos a noviembre de 2010 para proseguir explicando el segundo desbordamiento: si el primero fue de apertura y amplitud de discurso (de la oposición a la Ley Sinde a un cuestionamiento profundo del sistema democrático y de gestión del Estado), el segundo es una ampliación del primero -el discurso sobre el estado del Estado y la democracia se amplía e intensifica en algunos elementos centrales (por ejemplo la necesaria modificación de la Ley Electoral) adquiriendo un carácter más propositivo- producto de la concurrencia, de la confluencia con más actores. En concreto, de una transfusión de participantes:

Creo que ahí hubo una especie de mezclas. Gente de \#Nolesvotes que empezó a apoyar, y ellos [Democracia Real Ya], como que empezaron a ver qué íbamos juntos. (Ricardo Adolfo Galli, documental \#nolevotes\#democraciarealya).

\section{Coordinadora Ciudadana: ¿en el germen de Democracia Real Ya?}

El 27 de noviembre de 2010 en el Ateneo de Madrid, a las cinco de la tarde, tenía lugar un encuentro presencial de diversos agentes (entidades, algunas más definidas y constituidas y otras menos, y particulares): el acto fundacional de una plataforma ciudadana. Para sus integrantes, suponía el nacimiento de «[...] la mayor rebelión cívica de nuestra historia: la sociedad civil se une contra la casta política en defensa de la democracia, la libertad y la unidad de España». Según registra el portal que lo aloja fue filmado y editado por Rafael Alba Padilla, con fecha de subida del 28 de noviembre de $2010^{12}$ y el título «Congreso de la sociedad civil por una democracia real. Así empezó el 15M (seis meses antes)».

$\mathrm{El}$ análisis que va a presentarse en este apartado tiene como fuente nuclear de obtención de indicios empíricos el citado documental. Es obligado, por tanto, explicitar que se trata de una filmación editada, donde los discursos presentes en la reunión han sido seleccionados,

por la universidad de Santiago de Compostela, doctor en administración especializado en sistemas de la información por la Universidad de California y bloguero pionero.

Ricardo Adolfo Galli es profesor en Sistemas Operativos en la Universidad de las Islas Baleares, miembro de Free Software Foundation (FSF), expresidente del grupo de usuarios de software libre BULMA y coprogramador de un conocido sitio web de promoción de noticias a partir de la participación en una comunidad virtual.

12 Disponible en: http://www.youtube.com/watch?v=PWN8X4ZKLjo 
recortados y ordenados en función de criterios estéticos. Por tanto, aunque el documental tiene vocación de fidelidad, puede entorpecer la labor etnosociológica. Sin embargo, asumido el límite que comporta como fuente de datos empíricos, resulta muy interesante establecer el análisis tratando la narración como una autopresentación colectiva, donde el esfuerzo está puesto en lo que se considera fundamental, en lo que se quiere transmitir a nivel político: el manifiesto público.

En este sentido, se presenta a este encuentro en el Ateneo de Madrid como el inicio de Democracia Real Ya meses antes de que el manifiesto convocante se haga público.

Una informante que participó en la gestación del pequeño grupo que comienza a elaborar el manifiesto y que después es militante activa en la acampada de la Puerta del Sol (y en las estructuras organizativas del $15 \mathrm{M}$ madrileño postacampada) al ser preguntada al respecto expuso tajantemente que «hubieron algunos fachas que se quisieron sumar, pero nada tenemos que ver con ellos. Ellos no estaban».

Respecto de Enrique Dans (a pesar del propio testimonio de éste corroborado por otros participantes) afirma que, ni el soporte web para la página de la convocatoria estaban a su nombre -información que ofrecieron algunos portales ligados a la izquierda extraparlamentaria y que se presentaba como indicio de que la convocatoria estaba relacionada con el Tea Party estadounidense, ya que al parecer Dans pertenecía a esos circuitos políticos-, ni tenía constancia de que éste hubiera participado «al principio». Más bien, en su opinión, pertenecía al gran grupo de oportunistas (al que añadía a un par de participantes que se erigieron como «portavoces autorizados» por los medios de comunicación el mismo día 15 de mayo) que trataron de «hacer carrera» al amparo de la mediatización de la gran movilización.

Sin embargo, el 18 y 19 de mayo de 2013 tenían lugar unas jornadas, nuevamente en el Ateneo de Madrid, organizadas por la Asociación Democracia Real Ya, una escisión de la plataforma constituida en abril de $2012^{13}$. A estas jornadas de 2013, que utilizaban el lema «Democracia Real Ya. Toma la calle», estuvieron invitados, entre otros, portavoces del partido MoVimento 5 Stelle italiano, incluido Beppe Grillo.

Resulta muy interesante cómo la conexión con el acto de finales de finales de 2010 no solo se establece con el lugar y con que sea organizado por una escisión que precisamente constituye la apuesta institucional de Democracia Real Ya; sino que uno de los maestros de ceremonias de estas jornadas de 2013 es Mitoa Edjang Campos (del Proyecto Escáner -lucha por la eliminización de los escáneres en los aeropuertos-) quien también participó activamente en la primera asamblea en el Ateneo de Madrid de noviembre de 2010.

A partir de estos indicios - a pesar de la afirmación tajante de la informante que se ha presentado antes y más allá de las reticencias que podría suponer el planteamiento-, considero que se puede sostener que este bloque participó (de manera más o menos difusa, ciertamente) en la gestación de la convocatoria del 15 de mayo. Si bien es cierto que más adelante veremos la evolución del proceso y qué posiciones de este bloque quedaron definitivamente fuera del llamamiento del 15 de mayo del 2011.

13 Pueden verse las notas de prensa que recogerían las peleas internas entre DRY y ADRY, por ejemplo: «Democracia Real Ya, la asociación que impulsó el 15-M se parte en dos» [EL PAIS 23 de abril de 2012. Disponible en: http://politica.elpais.com/politica/2012/04/23/actualidad/1335212093_805436.html] o «Democracia Real Ya 'expulsa' a cinco de sus miembros» [Público, 24 de mayo de 2012. Disponible en: http://www.publico. es/431957/democracia-real-ya-expulsa-a-cinco-de-sus-miembros]. 
Volviendo al encuentro de la Coordinadora Ciudadana en el Ateneo de Madrid de finales de 2010, el espacio donde se desarrolla la asamblea está dispuesto en relación a una mesa principal sobre una tarima y sillas colocadas en el público. La sala está repleta de gente, alrededor de ochenta personas -las que no tienen asiento, asisten de pie en el fondo- mayoritariamente de mediana edad. Cuando quienes están en la parte dedicada al público toman la palabra, se colocan delante de la tarima sosteniendo un micrófono inalámbrico. En la mesa de conferencia están sentadas cuatro personas que hacen funciones organizativas y que, al parecer, han promovido el encuentro: Bernardo Rabassa Asenjo ${ }^{14}$, Jesús García Barcala (director de una empresa de consultoría en comunicación), Margarita Rabassa (diplomada por la ESIC: Business \& Marketing School, perteneciente al Club Liberal y colaboradora en medios digitales de la órbita liberal) y Juan Vicente Santacreu ${ }^{15}$. Meses más tarde, en la página web de la agrupación que lidera éste último, se publicará una entrada, firmada por el propio Santacreu, donde se culpabiliza a Jesús García Barcala y Alberto Medina ${ }^{16}$ de desmantelar y hundir el proyecto político que pretendía la plataforma y se anuncia, en consecuencia, la salida de Masby del proyecto político colectivo ${ }^{17}$. El nombre de Jesús García Barcala, Alberto Medina y Margarita Rabassa ya aparecía ligado a principios de noviembre de 2010 en un grupo coordinado en el portal Facebook bajo el nombre de Coordinadora Ciudadana, eran parte del grupo impulsor de la coordinadora que gestará la asamblea en el Ateneo de Madrid.

Las posiciones políticas que componen la plataforma se mueven desde el marco de la tradición del pensamiento liberal a la extrema derecha más reaccionaria, siendo el liberalismo, en su polo más democrático, el espacio político de confluencia, de encuentro de todos y todas las participantes. Es la posición ideológica capaz de cohesionar la plataforma que se está gestando, capaz de incluir a la extrema derecha y ciertas posiciones de centro, e incluso de centro-izquierda. Esto se concreta, en primer lugar, en la vocación de la mayoría de organizaciones que componen la plataforma ${ }^{18}$. En segundo, en la figura de Bernardo Rabassa Asenjo,

14 Periodista y director del periódico digital Periódico Liberal El reformista. Presidente honorario Club Liberal Español y presidente de la fundación FIECS, dependiente del Instituto Europeo para la Comunicación Social. Fue fundador del Partido Liberal en Mallorca y secretario y primer presidente de la Organización del Centro Democrático (que daría paso a la Unión de Centro Democrático).

15 Es el líder de la Plataforma Masby y candidato independiente por las listas del partido de extrema derecha España 2000 en la Comunidad Valenciana para las pasadas elecciones de mayo de 2011. Por su parte, Masby se autodefine como el Tea Party español (título que se disputa con otra asociación). Según explican en su página, el nombre se refiere al término anglosajón mass by que traducen como «pasar por encima de la masa». El discurso se centra fundamentalmente en: a) la defensa de la «unidad de España», sin más argumento que una lectura sesgada y falsa de la historia del territorio, que entronca con toda la tradición xenófoba concentrada en las personas migrantes, especialmente las de origen árabe; b) libertad, definida en torno a la abolición de la oficialización del multilingüismo -se habla de «lenguas tribales»-; y c) educación libre, también definida en función de la castellanización de las instituciones educativas y planes de estudio.

16 Militante del Partido Popular. Ha ostentado diversos cargos para el partido, en la actualidad es alcalde de Retortillo de Soria por el PP.

17 Resulta interesante ver el comunicado publicado en su página web con el título «Dando Caña Intereconomía Javier Algarra Masby España» donde puede apreciarse, además de los límites y orientación del levantamiento de sospechas, la violencia que se moviliza. [Disponible en: http://www.masaborreguera.com/videos/dando-cana-masby.html].

18 El colectivo de asociaciones que componen la coordinadora son: Aranjuez 1812, ARHOE, Círculo Liberal Español, Círculo Balear, Círculo Montalbán, Ciudadanos en la Red, Ciudadanos por la Democracia, Club Republicano Tres, Democracia Participativa, Decisión Ciudadana Democrática, España y Libertad, Hay que echar a Zapatero como sea, J.A.S.P., Liberales por el Cambio, Listas Abiertas, M.A.S. Jóvenes, Mayoría Democrática, MCRM, Movimiento 
que actúa como maestro de ceremonias y que goza de muchísimo prestigio en el grupo -la mayoría de participantes, cuando tienen la palabra, vuelven la cabeza a la mesa de conferencias buscando la mirada cómplice de Rabassa, o quizá su aprobación-. Y en tercer lugar, porque mayoritariamente los discursos recogidos en el documental (conviene recordar que es producto de un trabajo de edición) se sitúan en esta tradición de pensamiento - «Con menos Estado se podría vivir mejor, déjenos a la sociedad civil. No se empeñen en decirnos lo que es bueno o malo, que ya somos mayorcitos para decidirlo»; «Hay que ir a la reconquista de la democracia, no vale quedarse quietos. La democracia se ha perdido y como Pelayo, tenemos que reconquistarla desde dentro del aparato. No somos políticos de la casta, pero tenemos que entrar en el aparato. Si no, no tenemos ninguna opción»-, opacando, invisibilizando, o dando poco espacio a las posiciones más extremistas ${ }^{19}$.

Quedan recogidos también discursos claramente situados en el liberalismo economicista, esto es: la libertad y la democracia existen solo, y, solo si, es posible hacer buenos negocios, sin trabas administrativas. De manera más reducida, pero igualmente presentes, se encuentran discursos situados en la crítica a las políticas del PSOE. También encontramos el repertorio de la extrema derecha española suavizando la beligerancia que les suele acompañar y en una proporción reducida con respecto al espacio discursivo general. A pesar de que no se corresponde con la mayoría de las intervenciones, las ideas fuerza que lo estructuran (fundamentalmente el rechazo a la diversidad cultural de España, la diversidad histórica y la que resulta de las migraciones de las últimas décadas, y la oposición radical a las políticas del PSOE, el proyecto «Alianza de Civilizaciones», por ejemplo, parecen ser compartidas a la luz de los aplausos que reciben quienes los exponen.

Es importante señalar que, al igual que sucederá más adelante en las asambleas en las plazas, la sintonía entre los participantes puede - de hecho lo es- ser parcial. Se deben tener en cuenta, para poder comprender estas relaciones distanciándolas de lo estrambótico, las posibilidades de cohesión del habitus colectivo que se está conformando. Ya que las divisiones de la analogía según la cual se incorporan las disposiciones no son «ni exclusivas ni exhaustivas» (Bourdieu, 2000, 332 en Moreno Pestaña, 2004, 157) y pueden convivir en el mismo sistema de habitus disposiciones enfrentadas, antagónicas (Bourdieu, 2004, 204), haciendo que el habitus, en su esfuerzo por armonizarlas, no siempre articule las prácticas (los discursos) al uso de la lógica teórica. Si pensamos en ese agente como un cuerpo colectivo en construcción, podemos comprender cómo los discursos y las ideas fuerza (asentadas íntimamente en disposiciones ético-políticas) pueden ser parcialmente antagónicas, pero igualmente pueden convivir en el cuerpo colectivo, pudiendo activar resignificaciones simbólicas ${ }^{20}$. Aunque, al mismo tiempo, sin duda esa convivencia está

Democrático, Plataforma Cívica Ciudadana, Por una verdadera democracia, Rebelión Digital, Red Democrática, Plataforma Ciudadanos Descontentos Políticamente, Partido Libertario de España, Proyecto Escaner, RSCI, Social Party, UNES, Yo No Vote a ZP, Plataforma por la Regeneración Política de España, Centro Canario Independiente, Centro Democrático Andaluz, Movimiento Democrático de la Guardia Civil, Liberales Demócratas Centristas. A los que se les sumarían en el acto del Ateneo: Alternativa Liberal Social, España 2000, periódico El Reformista, Masby, Plataforma de las Clases Medias, P-Lib, Liberales demócratas centristas, Partido de los Mayores y Autónomos.

19 Basta observar lo editado que se encuentra el discurso de Santacreu en los dos fragmentos en los que aparece, apenas se intuye cuál es su postura.

20 En ese sentido, un análisis de los lemas y cánticos más coreados en las manifestaciones posteriores puede darnos pistas para rastrear los universos sociopolíticos (por tanto, las trayectorias vitales) ligados a diversos 
marcada por la lucha de los agentes, particulares o colectivos por instalar sus fundamentos, su visión, al cuerpo colectivo. Y no está exenta de nuevas entradas (que dependiendo de su fuerza, reordenan más o menos la conformación existente) o de salidas (al resultar insoportable la nueva formación). Además, la resonancia de algunas ideas fuerza (también en las formas organizacionales y en las herramientas de trabajo), en algunos casos, amortigua (a veces, temporalmente) la emergencia (a veces, consecuencia de un sesgo en la percepción) del enfrentamiento de otras.

Por otro lado, todo hace pensar que defender la «unidad» de España, la expulsión de las personas migrantes, la no negociación con ETA, el endurecimiento de las penas de la Ley del Menor o clamar contra la corrupción política, en principio, no está reñido con vindicar la naturaleza equilibrada del capital y la neutralidad divinizada de los mercados. Tampoco con una apuesta por la desaparición del Estado social, o con la defensa metafísica de las libertades individuales de los hombres blancos propietarios.

He querido dejar para el final los discursos enfocados a cohesionar el grupo: a pesar de que su presencia es casi anecdótica, en ellos reside toda la intención movilizadora, toda la fuerza aglutinadora de la convocatoria pública: «Unámonos, unámonos todos de verdad y quitémonos protagonismos, sea quien sea. Unamos un solo nombre y cacareémoslo»; «Si no contamos con todas aquellas personas que vienen de la izquierda, este movimiento no alcanzará sus objetivos: la democracia». Estas dos intervenciones son fundamentales porque marcan, de un lado, una brecha: la ruptura entre la dicotomía derechas/izquierdas que pretendía ya \#Nolesvotes y que será la bandera de Democracia Real Ya: una de las claves del éxito de la convocatoria. Y de otro, la idea de constituir un eslogan, un nombre, un paraguas aglutinador único: otra de las llaves: somos todo el mundo, «somos el 99\%».

Esta plataforma, o algunas de las personas que la componen, desembocarán en otro espacio mayor, del que según el documental serían parte, e incluso germen (o parte de

relatos sociales que conviven en el crisol simbólico. De «iEstas son nuestras armas! [exhibiendo las palmas de las manos abiertas]», símbolo de las movilizaciones ciudadanas (con masiva presencia de las cúpulas de casi todos los partidos políticos) tras el secuestro y asesinato anunciado de Miguel Ángel Blanco por ETA; a «¡El pueblo unido jamás será vencido!», coro de la composición musical del grupo Quilapayún para el momento de mayor asedio al Gobierno de la Unidad Popular que había llevado a Salvador Allende a la presidencia. Estos dos lemas, notablemente resignificados (pues se utilizan como aliento simbólico a la resistencia ante los desalojos forzados por parte de la policía) son coreados desde la primera manifestación y parecía que casi todo el mundo los conocía (no hacía falta nadie guiando con un megáfono). Conforme pasa el tiempo (sobre todo del verano en adelante) se van incorporando otros donde sí podemos identificar la fuente original porque presenciamos el proceso por el que pasa de ser un cántico coreado por unas diez, veinte o treinta personas a incorporarse al repertorio de cada acto y manifestación. Por ejemplo: «La democracia es una pocilga que está al servicio de los capitalistas. No tengas miedo, que no te callen. Es el momento de salir a la calle», incorporado al repertorio por militantes de un pequeño partido de izquierda extraparlamentaria. O «iDe Norte a Sur, de Este a Oeste, la lucha sigue: cueste lo que cueste!», incorporado por militantes de espacios anarquistas. Siguiendo la diferenciación de Randall Collins (2009, 207), en este último caso estaríamos ante símbolos unilaterales: adquiridos de otras personas en un proceso acumulativo en el tiempo conforme se ahondan las resonancias emocionales de ser miembro del grupo. Pero también encontramos desde los primeros días lemas como «Que no, que no, que no nos representan» o «No hay pan para tanto chorizo» que, junto con las resignificaciones señaladas más arriba, y junto con la asamblea de la plaza y la acampada, son, sin duda, nuevos símbolos que emergen del propio encuentro. Y siguiendo a Collins, esto es posible cuando «[...] el encuentro consigue crear un nuevo nivel de reflexión acerca de otras personas, o bien de comprensión mutua entre los participantes [...]» (Ob. cit.: 208). 
éste): Democracia Real Ya. Y ésta, comienza a relacionarse con otros colectivos, con otras plataformas y otras propuestas que circulaban ya en el ambiente cibernético ${ }^{21}$ :

Entonces lanzamos esa idea [la propuesta de \#Nolesvotes] y con la acogida que tuvo aquello empezaron a aparecer otros bloques por ahí. Creo que los primeros fueron la gente de Juventud Sin Futuro, que empezaron a escribir cosas, a ponerse de acuerdo [...] y ahí apareció la gente de Democracia Real Ya. Yo los conocí en una «redada» 22 de Medialab Prado ${ }^{23}$. Al día siguiente les mande un mail diciendo que si querían poner mi adhesión a título individual en su página, que yo encantado. (Enrique Dans, documental nolevotes\#democraciarealya).

Quedan todavía algunos hechos que narrar, queda también explicar cómo una convocatoria que tiene su embrión en la oposición a una forma de regular la forma de compartir y difundir conocimiento o información en la red virtual (y todo ello, como decía, enmarcado en una postura alineada con el liberalismo económico -el Estado no debe intervenir, la oferta y la demanda ajustarán las relaciones comerciales en Internet, y pondrán en su lugar a cada cual-) que se fusiona (o es alimentada) con una plataforma situada también en el liberalismo (más o menos democrático, más o menos economicista, más o menos receptor de las reivindicaciones de la extrema derecha) convoca bajo el sublema «no somos mercancía en manos de políticos y banqueros» y dos meses después, el 19 de Julio de 2011,

21 Antolín (2014) indica la existencia del grupo Estado del Malestar como parte de algo que nombra como plataforma de grupos promovilización». Según explica la autora, Estado del Malestar habría emergido desde el contacto entre seis personas a partir de una conversación en un «evento» del portal Facebook.

Estado del Malestar abría el nuevo ciclo movilizador el 11 de febrero de 2011 con una pequeña concentración en la Puerta del Sol a la que habrían asistido poco más de 15 personas: amistades y familia de las personas convocantes. Sin embargo, a partir de la creación de un grupo abierto en Facebook y la publicitación de la existencia del mismo por parte de Andreu Jaume, impulsor de la petición de dimisión para la ministra González Sinde, en el mensaje de agradecimiento a las más de 26.000 personas que lo firmaron, disparó las solicitudes de ingreso pasando de 300 a 3000 en veinticuatro horas (cuando trasladen el grupo abierto al formato página, en la misma plataforma Facebook, llegarán a tener 17.000 seguidores). Además se ramificaron y multiplicaron los grupos locales de Estado del Malestar por toda la geografía nacional. Para el 16 de febrero de 2011, apenas cinco días después de la pequeña concentración en la Puerta del Sol, existían grupos en Alicante, Barcelona, Cádiz, Castellón, Granada, Lugo, Mallorca, Murcia, Salamanca, Santander, Sevilla, Tarragona, Valencia y Vigo. Entre las normas de participación del grupo abierto en el portal Facebook que crean sus promotoras se encuentran enormes resonancias con los planteamientos que se vienen exponiendo pero se pueden ver, quizá más nítidamente, algunas condiciones de posibilidad para la participación en el 15M: «No asociación del grupo a ningún partido político, sindicato u organización; Movilizaciones ciudadanas como objetivo principal del grupo; Responsabilidad individual de los miembros sobre lo que publicaran, como representantes únicamente de ellos mismos; Petición expresa de no hacer uso de la plataforma para hacer publicidad de partidos políticos y sindicatos o de otros grupos de manera interesada; rechazo de la violencia y apoyo a manifestaciones pacíficas y creativas».

22 La \#redada es un formato de actividad en la que se entrevista a un invitado in situ combinado con una transmisión in streaming (en directo mediante una cámara web, que permite la interacción del espectador) y una línea de comunicación mediante el portal Twitter (que permite seguir el hilo de una conversación y participar en ella, si se desea, con mensajes cortos y rápidos).

23 Un espacio para producción, investigación y difusión de la cultura digital en relación al mundo artístico del Área de Las Artes del Ayuntamiento de Madrid. 
llama a otra movilización con el lema: «No al pacto del euro. Europa para los ciudadanos, no para los mercados ${ }^{24} \gg$. Aquí entra en juego Juventud Sin Futuro.

\section{Juventud Sin Futuro. Tercer desbordamiento: el viaje hacia la izquierda}

El siete de abril de 2011 se desarrollaron manifestaciones principalmente en Madrid (núcleo de la protesta) y Barcelona bajo el lema «Juventud Sin Futuro: sin casa, sin curro, sin pensión, sin miedo». La convocatoria venía impulsada desde la coordinación de diversos actores universitarios, asociaciones y centros sindicales, conformando una plataforma que comenzaba a trabajar bajo el sistema de comisiones y asambleas y que se extendía al resto del territorio español fundamentalmente a través de las redes sociales.

Más de 2.000 personas según los medios, 5.000 según la organización, participaron en una manifestación al grito de: «iAquí está la juventud precaria!».

El manifiesto ${ }^{25}$ que llamaba a la movilización fue firmado por más de 5.400 personas a la fecha y hora de la manifestación (las adhesiones rozan las 9.700 en diciembre de 2011 -última fecha de consulta-). Entre los y las firmantes se encontraban personajes públicos del mundo de las letras, el pensamiento, la investigación y de la cultura en general, como Jorge Riechmann, Félix Ovejero, Marcos Ana, Santiago Alba Rico, Salvador López Arnal, Javier Gallego Garrido, Almudena Grandes, Guillermo Toledo, Jaime Pastor, Luis García Montero, Roberto Gañan de Ska-p, Enrique Santiago Romero, Ramón Sánchez Tabarés, Antonio Domènech, Antonio Izquierdo Escribano, Francisco Fernández Buey, Vera Sacristán, Luis Enrique Alonso o Amelia Valcárcel. Fue firmado fundamentalmente por agentes universitarios: profesorado, becarias y becarios de investigación, personal técnico, alumnado y personal de administración y servicios y personas en situación de desempleo -egresados universitarios mayoritariamente-. Aunque también incluyó muchas adhesiones del mundo de las artes visuales, el diseño, el periodismo y las humanidades. De manera casi marginal, se encontraban también adhesiones de empleadas del hogar, jubilados y jubiladas, obreros y obreras y algún que otro policía. Las organizaciones que secundaron el manifiesto pertenecen a un universo simbólico y a un espectro político más o menos convergente: organizaciones estudiantiles, secciones sindicales de CGT y UGT, delegaciones del PSOE, IU, PCE, organizaciones LGTB, diversas delegaciones de ATTAC, asociaciones vecinales y ONGds ${ }^{26}$.

El manifiesto de Juventud Sin Futuro partía de una toma de posición respecto a la crisis económica que está viviendo el país, vindicando una gestión de la situación económica contraria a la adoptada por el Gobierno del PSOE. Una oposición a las medidas adoptadas por el Ejecutivo que entroncaba con toda la tradición de izquierda estatista, defensora del Estado social. En concreto a aquellas condensadas en lo que se considera una «política de recortes sociales».

24 No puedo explicarlo aquí detenidamente, pero ese sublema tiene relación con la entrada posterior de ATTAC (firmante de la convocatoria de Juventud Sin Futuro) al interior de DRY (se debe señalar que, en la ciudad donde se ha desarrollado la etnografía, DRY y la asamblea de la acampada se mantuvieron independientes y medio una no muy buena relación). Según contaba un miembro de ATTAC en la ciudad donde he desarrollado la etnografía, a mediados de julio eran ellos la única organización a la que se le había «consentido» la participación al interior de la plataforma. Parece claro que buena parte de la transformación o concreción ideológica de DRY durante el verano de 2011 es debida a este ingreso.

25 Disponible en: http://juventudsinfuturo.net/manifiesto-jsf/

26 Listado completo de firmantes disponible en: https://docs.google.com/spreadsheet/ccc?authkey=CNC8oNwG\&hl=en US\&key=0Aq5mTZ_CWhNUdGlySIJWcXM0UzJOM2E0eUFUWIFuY3c\&rm=full\&authkey=CNC8oNwG\#gid=0 
Como expuse más arriba, esta convocatoria nació del entorno universitario y es desde ahí desde donde se articuló un discurso que, sin embargo, tenía vocación generalista. No se trata de una defensa de las instituciones universitarias o de los agentes que la componen, sino de la juventud: de una generación, y con ésta, del futuro del país.

[...] nos dirigimos a la opinión pública para mostrar nuestro desacuerdo con la política de recortes sociales del Gobierno, y la consecuencia más grave y con mayor impacto en el futuro que estas medidas representan: la juventud más preparada de nuestra historia vivirá peor que sus padres.

Tres son los elementos que condensan la «agresión contra el colectivo juvenil en un escenario de crisis capitalista» plasmado en una tasa de paro del 40\%: la Reforma laboral, la Reforma del sistema de pensiones y la mercantilización de la educación pública.

Los dos primeros elementos eran contingentes, esto es, hacían referencia a decisiones políticas tomadas en la coyuntura de crisis económica del país (o al menos así habían sido presentadas: «medidas anticrisis»). El tercero hace referencia a un proceso dilatado en el que han colaborado los últimos gobiernos nacionales y regionales que vienen afianzando el establecimiento de relaciones laborales desreguladas en el seno de la universidad y entregando ciertas zonas de gestión a empresas privadas. El manifiesto continuaba señalando a las personas responsables: las élites económicas y políticas que no han hecho más que «socializar las pérdidas» de un entramado creado por ellos mismos. Y culmina haciendo un llamamiento a «recuperar nuestra capacidad para ser actores de un motor de cambio».

Hasta el momento he presentado lo que considero un triple proceso de desbordamiento del germen de la convocatoria del 15 de mayo.

Quiero insistir en esta idea de un proceso de desbordamiento de discurso y de agentes para señalar que, a pesar de que el lema existía con anterioridad a la trasfusión de participantes que provenían del espacio Juventud Sin Futuro (como indica el hecho de que Democracia Real Ya firmara el manifiesto el 30 de marzo de 2011, lo que indica su existencia de manera paralela), existen diversos elementos que hacen pensar que precisamente en esa trasfusión de Juventud Sin Futuro a la plataforma Democracia Real Ya es donde reside la clave para comprender lo que he denominado «viaje hacia la izquierda». Esto es, la clave para comprender cómo una plataforma que emerge de un espectro social que se mueve entre posiciones del liberalismo democrático, liberalismo económico e incluso de extrema derecha, termina presentando un discurso capaz de movilizar y acoger en la convocatoria hasta a la izquierda extraparlamentaria (que se movilizó con recelo respecto del lenguaje del manifiesto pero acudió masivamente).

Esta trasfusión resultará fundamental y determinará sobremanera el resultado final: las ideas fuerza (y la estética) del material que llamaba a la manifestación y el origen político de los apoyos recibidos en el universo de movimientos sociales de izquierda que dio confianza a quienes recelaban de un manifiesto que había evacuado completamente cualquier resonancia con el imaginario simbólico y conceptual del marxismo.

Detengámonos un instante para estudiar los lugares de posible encuentro de los tres bloques. Para ello presentaré un cuadro resumen que recoge las ideas fuerza de cada uno 
de ellos, estableciendo los encuentros y desencuentros y finalmente qué de todo aquello quedó recogido en el comunicado que llamaba a la movilización el 15 de mayo.

\begin{tabular}{|c|c|c|}
\hline \#NO LES VOTES & COORDINADORA CIUDADANA & JUVENTUD SIN FUTURO \\
\hline $\begin{array}{c}\text { Corrupción del sistema político } \\
\text { encarnado en PP, PSOE, CIU y PNV. }\end{array}$ & $\begin{array}{l}\text { Corrupción (que incluye terrorismo } \\
\text { de Estado) del sistema político } \\
\text { encarnado en el PSOE. }\end{array}$ & $\begin{array}{c}\text { Análisis crítico de las medidas } \\
\text { «anticrisis» adoptadas por el } \\
\text { Gobierno del PSOE. }\end{array}$ \\
\hline \multicolumn{3}{|l|}{ Bipartidismo (turnismo). } \\
\hline Clientelismo y partido-aparato. & Clientelismo y partido-aparato. & \\
\hline $\begin{array}{c}\text { Desconexión entre representantes y } \\
\text { representadas/os. }\end{array}$ & $\begin{array}{c}\text { Desconexión entre representantes y } \\
\text { representadas/os. }\end{array}$ & $\begin{array}{l}\text { Desconexión entre representantes y } \\
\text { representadas/os. }\end{array}$ \\
\hline $\begin{array}{l}\text { Elitización (profesionalización) de los } \\
\text { espacios de decisión política. }\end{array}$ & $\begin{array}{c}\text { Elitización (profesionalización) de los } \\
\text { espacios de decisión política. }\end{array}$ & $\begin{array}{c}\text { Elitización (profesionalización) de los } \\
\text { espacios de decisión política. }\end{array}$ \\
\hline \multicolumn{3}{|l|}{$\begin{array}{l}\text { Canales para participación ciudadana } \\
\text { cerrados. }\end{array}$} \\
\hline \multicolumn{3}{|l|}{$\begin{array}{l}\text { Ley electoral: falsa representatividad } \\
\text { de la pluralidad ideológica. }\end{array}$} \\
\hline \multirow[t]{4}{*}{$\begin{array}{l}\text { Llamada a la toma de } \\
\text { responsabilidades ciudadanas y a la } \\
\text { toma de conciencia. }\end{array}$} & $\begin{array}{l}\text { Llamada a la toma de } \\
\text { responsabilidades ciudadanas y a la } \\
\text { toma de conciencia. }\end{array}$ & $\begin{array}{l}\text { Llamada a la toma de } \\
\text { responsabilidades ciudadanas y a la } \\
\text { toma de conciencia. }\end{array}$ \\
\hline & $\begin{array}{l}\text { Vindicación de un necesario } \\
\text { adelgazamiento del Estado. }\end{array}$ & $\begin{array}{l}\text { Anticapitalismo. Oposición a la } \\
\text { perspectiva economicista neoliberal. } \\
\text { Defensa del Estado social. }\end{array}$ \\
\hline & $\begin{array}{l}\text { Estado centralista. Oposición al } \\
\text { proyecto de autonomías. Y a la } \\
\text { subvención por parte del Estado de } \\
\text { organizaciones sindicales. }\end{array}$ & \\
\hline & & $\begin{array}{l}\text { Análisis de las repercusiones de la } \\
\text { crisis para la juventud española. }\end{array}$ \\
\hline $\begin{array}{l}\text { Vocación aperturista, llamamiento } \\
\text { al encuentro más allá de la opción } \\
\text { política. }\end{array}$ & $\begin{array}{l}\text { Vocación aperturista, llamamiento } \\
\text { al encuentro más allá de la opción } \\
\text { política. }\end{array}$ & $\begin{array}{l}\text { Vocación aperturista, muestra de } \\
\text { pluralidad }^{27} \text {. }\end{array}$ \\
\hline $\begin{array}{l}\text { La estrategia militante se enmarca } \\
\text { en los espacios de participación } \\
\text { institucionalizados. }\end{array}$ & $\begin{array}{l}\text { La estrategia militante se enmarca } \\
\text { en los espacios de participación } \\
\text { institucionalizados. }\end{array}$ & $\begin{array}{l}\text { La estrategia militante se enmarca } \\
\text { fuera de los espacios de participación } \\
\text { institucionalizados. }\end{array}$ \\
\hline
\end{tabular}

LEYENDA: En granate está indicado aquello sobre lo que a nivel ideológico no existe consenso, pero que tiene la posibilidad de traducirse en conceptos o prácticas integradoras. Por ejemplo el concepto PPSOE, o Rubaljoy empleado masivamente para señalar que ambos partidos gobiernan de la misma manera y con idénticos intereses: sostener sus privilegios. O acudir al acto de investidura de los ayuntamientos para manifestar el descontento con la gestión política del país, al grito de «iQué no, qué no, qué no nos representan!». En verde están indicadas aquellas ideas fuerza en las que convergen. En naranja aquello que he considerado divergencias, o incluso ideas fuerza abiertamente enfrentadas.

27 Si bien la postura no es idéntica, e incluso relativamente opuesta, sí considero que sobrevuela una voluntad de ir más allá de la alineación política más específica. Los apoyos se buscan en el universo simbólico de izquierda, pero de una izquierda democrática, sosegada e intelectualizada. Busca movilizar a la «juventud» y entiendo que es un constructo relativamente flexible en cuanto a las posibilidades de adscripción. 
Podrán reconocerse entre las ideas fuerzas convergentes buena parte del argumentario y centros de interés del 15M; sobre los disensos, los lugares de discusión más frecuentes en las asambleas de las primeras semanas y, finalmente, sobre lo que no hay acuerdo pero se pudo converger en algunas articulaciones prácticas que operaron sobre una base de ambigüedad. Sin duda, en este cuadro se encuentran indicios importantes que anuncian los marcos acotados de encuentro y el tamaño de la brecha que atravesaba los desencuentros.

\section{Cuarto desbordamiento: el manifiesto se pone en circulación}

Finalmente Democracia Real Ya lanzó un manifiesto ${ }^{28}$ a la ciudadanía, fundamentalmente difundido por la red virtual. Paralelamente comenzó a sondear posibles adhesiones de colectivos políticos, culturales, humanitarios etc., que se sumarán a la convocatoria ${ }^{29}$.

Una semana antes de la cita nacional, se compartían las convocatorias por ciudades en el muro de Facebook, se retwitteaba y se reenviaban continuamente correos con información al respecto (que en muchos casos incluían la versión digital del libro del nonagenario Stéphane Hessel jIndignaos!).

El manifiesto único condensará la idea de apertura del espacio social: «somos gente como tú» que trata de representar a las «personas normales y corrientes»; por oposición, se intuye, a las élites económicas y políticas (de ahí la especificación de tareas o labores cotidianas que implican esfuerzo y angustia, incluyendo «buscar trabajo»). Y apertura también ideológica: se sustituye la oposición izquierda/derecha para situarse en un continuo donde «unos nos consideramos más progresistas, otros más conservadores». La cohesión de un colectivo diverso se condensa en: «todos estamos preocupados e indignados por el panorama político, económico y social que vemos a nuestro alrededor. Por la corrupción de los políticos, empresarios, banqueros... Por la indefensión del ciudadano de a pie».

El texto viene a reivindicar una sociedad que avanza en igualdad, solidaria, donde haya un libre acceso a la cultura, una lógica productiva sostenible ecológicamente, un desarrollo para el bienestar y la felicidad de ciudadanía. En ese sentido, se establecen ciertos derechos considerados como básicos que deben estar cubiertos por una sociedad -no queda claro, porque no se dice, si debe ser el Estado quien asuma esa responsabilidad, o por el contrario será producto del supuesto equilibrio de los mercados y la iniciativa privada-: «derecho a la vivienda, al trabajo, a la cultura, a la salud, a la educación, a la participación política, al libre desarrollo personal, y derecho al consumo de los bienes necesarios para una vida sana y feliz».

La fórmula democrática, se explicará, está desvirtuada. Se utiliza para la argumentación la fuerza simbólica de la definición etimológica y se ofrecen unas conclusiones sobre el estado de la cuestión de la representación política muy negativas («en este país la mayor parte de la clase política ni siquiera nos escucha. Sus funciones deberían ser la de llevar nuestra voz a las instituciones, facilitando la participación política ciudadana mediante cauces directos y procurando el mayor beneficio para el grueso de la sociedad, no la de enriquecerse y medrar

28 Disponible en: http://www.democraciarealya.es/manifiesto-comun/

29 Es imposible reproducir aquí el listado de organizaciones, grupos de facebook, personas a título individual que componen el grupo de adhesiones. Destacan por ser asociaciones con larga trayectoria y amplitud de marco de acción 5 delegaciones de Ecologistas en Acción, 11 de ATTAC, 3 de la Asociación Pro Derechos Humanos de Andalucía. Listado completo disponible en: http://www.democraciarealya.es/adhesiones/. 
a nuestra costa, atendiendo tan solo a los dictados de los grandes poderes económicos»), señalando al partido en el Gobierno y al principal partido de la oposición («PPSOE» como si fuera un solo partido) responsables de la debacle democrática. El «funcionamiento del sistema económico y gubernamental» son el principal obstáculo para el «progreso de la humanidad», ya que sostiene a unos pocos y «bloquea la maquinaria social en una espiral que se consume a sí misma enriqueciendo a unos pocos y sumiendo en la pobreza y la escasez al resto». Además, continúa el manifiesto, tras la lógica de la acumulación económica, no hay más que despilfarro de recursos medioambientales que generan desempleo y «consumidores infelices».

La oposición a la lógica economicista capitalista se concreta en el penúltimo párrafo: «Si como sociedad aprendemos a no fiar nuestro futuro a una abstracta rentabilidad económica que nunca redunda en beneficio de la mayoría, podremos eliminar los abusos y carencias que todos sufrimos». Que dará el pie para, en el último punto, llamar a una revolución ética, una revolución que supone restablecer el concepto de ciudadanía que ha sido reemplazado por el de consumidor.

La propaganda preparada para la manifestación presentaba una estética exageradamente similar a la Juventud Sin Futuro (y a la de V de vivienda, una convocatoria de 2009 que también utilizó la acampada como estrategia movilizadora y espacio anterior de movilización de varias personas participantes en la gestación de DRY), lo que invita a pensar en un mismo grupo de diseño, en criterios estéticos semejantes o en la toma de modelo del primero para diseñar el segundo y el tercero.
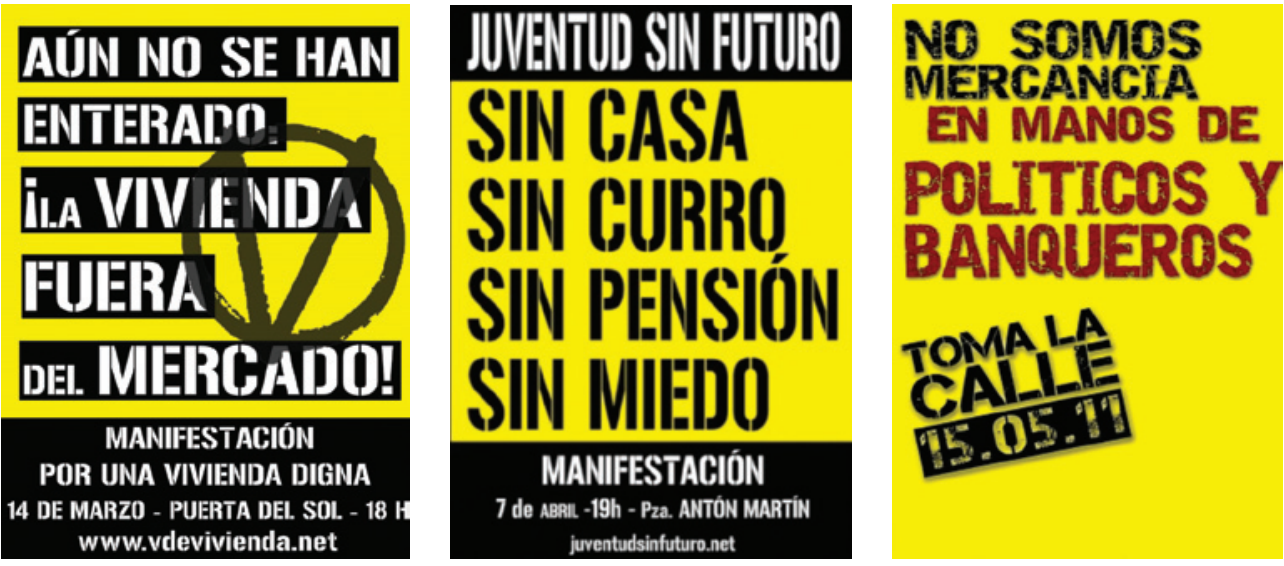

\section{Conclusiones}

Podemos intuir de qué manera se irán tejiendo las relaciones y sobre qué temas se evitará enfocar el discurso si -y esta es una idea que estructura, lo veíamos más arriba, los tres manifiestos- se quiere aglutinar y movilizar al máximo de personas posible. También las salidas, que sin duda debieron darse, de las posiciones próximas a la extrema derecha, a la luz del comunicado final de Democracia Real Ya.

Si bien he presentado a \#Nolesvotes, Coordinadora Ciudadana y Juventud Sin Futuro como espacios colectivos alimentadores de participantes para la protoestructura de la 
convocatoria, es necesario advertir que desde el momento en que la convocatoria se hizo pública y se habilitó un espacio virtual para la difusión y el reclutamiento de nuevos integrantes -además de las adhesiones y nuevos ingresos a través de organizaciones sociales-, el espacio colectivo de confluencia fue desbordado sucesivamente. Además, desbordado de una manera invisible, casi individual (puesto que la mayoría de personas acceden a través del contacto directo enviando un correo electrónico y suscribiéndose a los grupos de trabajo; o mediante el contacto a través de amistades) dando como resultado células de Democracia Real Ya en multitud de ciudades y con relativa diversidad y autonomía. En la ciudad donde he concentrado el estudio de caso, las personas que componían el grupo motor de la convocatoria invitaron a una reunión de presentación de la propuesta de movilización a un variado y amplio espectro de organizaciones. La propuesta era clara: sin banderas, ni siglas. El manifiesto estaba ya redactado: funcionaba como elemento de presentación y al que adherirse. El material gráfico también.

Quiero insistir en el despliegue de organizaciones que sostuvieron, participaron y/o publicitaron la movilización, contradiciendo la idea general de que es un movimiento espontáneo. Ciertamente esa idea es constantemente reforzada por los propios participantes ya que en el esfuerzo amplificador de la protesta se apuesta por reivindicar que no hay ninguna organización que la sustenta como una forma de evitar posibles rechazos. Sin embargo, conviene señalar que, al mismo tiempo, esta circunstancia se acomoda muy bien a lo que Franck Poupeau (2007) considera un sesgo en la mirada analítica sustentado en una visión miserabilista de los dominados (extensible, en el caso que nos convoca, a los movimientos populares) que hace interpretar como espontáneas movilizaciones que, si bien se dan en momentos históricos extraordinarios o contienen ciertos elementos de espontaneidad militante, se sostienen sobre redes de relaciones diacrónicas y esfuerzos de organizaciones sociales invisibilizados ${ }^{30}$.

Parece, más bien, que nos encontramos ante un movimiento popular enraizado sobre redes de relaciones colectivas, para el que los recursos militantes y el capital simbólico de los movimientos sociales locales resultaron muy importantes, y sometido a sucesivos desbordes que fueron posibles gracias a su propia estructura funcional y a la búsqueda de inclusividad.

Sin duda queda abierta para una posterior investigación, que deberá entenderse como un trabajo colectivo y acumulativo, la tarea de afinar hasta lograr determinar con más exactitud el lugar y el papel que tuvieron todos estos cuerpos colectivos. Así como rescatar a aquellos que, podría suceder, estuvieron presentes y no he dado debida cuenta de ellos.

\section{Bibliografía}

Antolín, V., «En los orígenes del Movimiento Indignado en España. Estado del Malestar: de Facebook a Sol, un estudio de caso», Congreso Tres Años de Indignación: la emergencia de nuevos sujetos sociopolíticos, UNED, 2014.

Bertaux, D., Los relatos de vida: perspectiva etnosociológica, Barcelona, Bellaterra, 2005. Bourdieu, P., Propos sur le Champ Politique, Lyon: Presses Universitaires de Lyon, 2000. - El baile de los solteros, Barcelona, Anagrama, 2004.

$30 \mathrm{Al}$ respecto, ver el trabajo de Poupeau sobre la llamada Guerra del agua en Bolivia (Poupeau, 2007, 58-61). 
- Sur l'État. Cours au Collège de France (1989-1992), en P. Champagne, R. Lenoir, F. Poupeau y M.C. Rivière (eds.), París, Raisons d’agir/Senil, 2012.

Bourdieu, P. (dir.) La miseria del mundo, Buenos Aires, Fondo de Cultura Económica, 2010 [1993].

Bourdieu, P. y L. Wacquant, Una invitación a la sociología refexiva, Madrid, Siglo XXI, 2005.

Collins, R., Cadenas de rituales de interacción, Madrid, Anthropos, 2009.

Francisco, A. de, Ciudadanía y democracia. Un enfoque republicano, Madrid, Los Libros de la Catarata, 2007.

Gunther, R., «Leyes electorales, sistemas de partidos y élites: el caso español», Revista Española de Investigaciones Sociológicas (47), pp.73-106, 1989.

Mathieu, L., Comment lutter? Sociologie et mouvements sociaux, Paris, Textuel, 2004.

- (2005): «L'espace des mouvements sociaux», Politix, (77), pp. 131-151, 2007.

Mauger, G., «Pour une politique réfexive du mouvement social», en P. Cours y M. Vakaloulis (dirs.), Les mobilisations collectives: une controverse sociologique, París, PUF, 2003.

- Repères pour résister à l'ideologie dominante, Bellecombe-en-Bauges, Éditions du Croquant, 2013.

Melucci, A., «Las Teorías de los Movimientos Sociales», Estudios Políticos, (4-5, 4-1), pp. 92-101, 1985.

Moreno Pestaña, J. L. «Cuerpo, género y clase en Pierre Bourdieu», en L. E. Alonso, E. Martín Criado y J. L. Moreno Pestaña (eds.), Pierre Bourdieu: las herramientas del sociólogo, Madrid, Fundamentos, 2004.

Ortí, A., «La apertura y el enfoque cualitativo o estructural: la entrevista abierta semidirectiva y la discusión de grupo», en M. García Ferrando, F. Alvira y J. Ibáñez (comp.), El análisis de la realidad social. Métodos y técnicas de investigación, Madrid, Alianza Editorial, 1986.

Péchu, C., «Les générations militantes à droit au logement», Revue française de science politique (51), pp. 73-103, 2001.

Poliak, P., «Attac. Aux frontières du champ politique», en B. Geay y L. Willemez, Pour une gauche de gauche, Bellecombe-en-Bauges, Éditions du Croquant, 2008.

Poupeau, F., Dominación y Movilizaciones. Estudios sociológicos sobre el capital militante y el capital escolar, Córdoba, Ferreyra Editor, 2007. 